Review Article

\title{
Acute Appendicitis
}

\author{
Daibo Kojima a,*, Ari Leppäniemi b, Suguru Hasegawa c \\ a Department of Surgery, Fukuoka University Chikushi Hospital. Fukuoka, Japan \\ ${ }^{b}$ Division of Gastrointestinal Surgery, Helsinki University Central Hospital, Helsinki, Finland \\ ${ }^{c}$ Department of Gastroenterological Surgery, Fukuoka University School of Medicine, Fukuoka, Japan
}

\section{Article history:}

Received: January 29, 2019

Accepted: July 11, 2019

\author{
*Corresponding Author: \\ Daibo Kojima \\ Department of Surgery, Fukuoka \\ University Chikushi Hospital, \\ Fukuoka, Japan \\ E-mail: dkojima@fukuoka-u.ac.jp \\ ORCID \\ https://orcid.org/0000-0001-5388-8320
}

\begin{abstract}
Acute appendicitis (AA) is one of the most common causes of acute abdominal pain, which can progress to perforation of the appendix and peritonitis. Recently, AA has been classified into uncomplicated (nonperforated, no phlegmon) or complicated (abscess, perforation, phlegmon) appendicitis, for an appropriate initial treatment. With respect to surgical treatment of AA, laparoscopic surgery has been widely accepted worldwide as a safe and feasible first-line treatment. Over the last decade, nonoperative treatment has been proposed as an alternative to surgery in uncomplicated $A A$, and has also played an important role in the management of complicated AA. AA is also the most common cause for abdominal surgery during pregnancy, though an accurate diagnosis of AA during pregnancy is challenging. In this review, the topics being discussed include: 1) Non-operative management for uncomplicated AA, 2) Management for AA in pregnancy, 3) Management for complicated appendicitis (especially immediate laparoscopic surgery for appendiceal abscess), 4) Appendiceal neoplasms related to complicated AA.
\end{abstract}

Keywords: appendicitis, appendiceal neoplasms, laparoscopic surgery, pregnancy

\section{Non-operative Management for Uncomplicated Acute Appendicitis}

Appendectomy has been the gold standard surgical treatment for $\mathrm{AA}$, on the basis that without surgical intervention the condition progresses to perforated appendicitis. Antibiotic therapy has been proposed as an alternative to an appendectomy, in uncomplicated cases. In order to elucidate the role of non-operative treatment of uncomplicated appendicitis, Varadhan et al [1] performed a meta-analysis that included 4 randomized controlled trials with a total of 900 patients (470 patients received antibiotic treatment and 430 underwent an appendectomy) [1]. The antibiotic treatment of appendicitis had a $63 \%$ success rate at 1 year, a lower complication rate, and a relative risk (RR) reduction of $31 \%$ compared with appendectomy treatment for appendicitis $(\mathrm{RR}=0.69, p=0.004)$. Moreover, if the studies with crossover of patients between antibiotic and surgical treatment were excluded from the analysis, the reduction in risk of recurrent appendicitis was higher (39\%), and RR was lower (0.61, $p=0.02$ ). The analysis did not observe significant differences in treatment efficacy, length of stay, or risk of developing complicated appendicitis. Thus, the meta-analysis provides evidence that antibiotic treatment is associated with reduced risk of complications, and no increased risk of perforation in patients having a delayed appendectomy after antibiotic failure. Although about $20 \%$ of patients who were treated with antibiotics also had an appendectomy due to recurrence of symptoms, and of these about 1 in 5 had complicated appendicitis. The authors concluded that a careful "wait, watch, and treat" policy may be adopted in those patients considered to have uncomplicated appendicitis. When a diagnosis cannot be made definitively, the correct diagnosis, rather than an early appendectomy is more important. 


\section{Spontaneous resolution of early uncomplicated appendici- tis may be common}

What is the natural course of AA? It has not been reported whether recovery from uncomplicated AA is the result of antibiotic therapy or natural clinical remission. Is antibiotic therapy required for all patients with suspected uncomplicated AA? A prospective randomized study in 2017 by Park et al [2] reported the first randomized trial to compare the outcome of a non-antibiotic management strategy with antibiotic therapy in uncomplicated AA. In this study, 245 patients with computerized tomography-verified uncomplicated simple $\mathrm{AA}$, were randomized into management with a non-antibiotic regimen with supportive care, or a 4-day course of antibiotics with supportive care [2]. Patients who had more than 11 mm diameter appendix, appendicolith, extraluminal gas, intraperitoneal fluid or suspected abscess (mass) formation were excluded. Patients who were pregnant were also excluded. The primary endpoint was the rate of total treatment failure, which was defined as initial treatment failure within 1 month, and recurrence of appendicitis during the follow-up period (a median of 19 months). For those patients randomized into the non-antibiotics group, the mean duration of hospital stay was shorter (3.1 days versus 3.7 days; $p<0.001$ ) and the medical costs were lower $(€ 1,181$ versus $€ 1,348$; $p<0.001$ ) than the antibiotic therapy patient group. There was no difference in total treatment failure rate between the 2 groups: 29 of 124 (23.4\%) in the non-antibiotic group and 25 of 121 $(20.7 \%)$ in the antibiotic therapy group ( $p=0.609)$. Eighteen patients ( 9 in each group) had initial treatment failure, 15 of whom underwent an appendectomy and 3 received additional antibiotics. Thirty-six patients (20 in the non-antibiotic group, 16 in the antibiotic therapy group) experienced recurrence, of whom 30 underwent an appendectomy and 6 received further antibiotics. An appendiceal diameter between $6 \mathrm{~mm}$ to $11 \mathrm{~mm}$, without complications can be considered as simple AA which has mild inflammation that may resolve spontaneously. The inflammation caused by AA may contribute to the resolution of this condition, and antibiotic therapy may not be necessary. Special attention should be given to the fact that 13 cases in this study had perforated appendicitis after the success of the initial treatment, and 1 patient had cystadenoma (even though their appendiceal diameter was within $11 \mathrm{~mm}$ ). The process of "active observation" is really important in such cases.

\section{There is no role for non-operative management of uncom- plicated AA in pregnant women}

Recent evidence to support the advice for the use of antibiotics alone in the treatment of $\mathrm{AA}$, has not been extended to pregnant patients. During pregnancy there is a higher rate of peritonitis, fetal demise shock, and venous thrombosis compared with operative management. Therefore, there is no role for non-operative management for uncomplicated AA in pregnant women. AA is the most common cause of acute abdominal pain during pregnancy, and accounts for $25 \%$ of non-obstetric surgery during pregnancy, and has an incidence of $1 / 500$ to $1 / 2,000$ among all pregnancies [3]. Diagnosis of AA during pregnancy is challenging. Ultrasound imaging is the most commonly used and easily performed diagnostic tool for AA in pregnancy. However, changes in the location of the appendix and low sensitivity rates of ultrasound imaging may cause diagnostic delays that may lead to more complications during pregnancy compared with non-pregnant women. The difficulty in the diagnosis of AA during pregnancy is associated with the high rate of negative appendectomies [4]. There were concerns that negative appendectomies were linked with fetal loss [5]. In 2012, Wilasrusmee et al [5] performed a meta-analysis including 11 studies, with a total of 3,415 women (599 in laparoscopic and 2,816 in open group) and showed an increase in fetal loss rate with a laparoscopic approach, compared with open appendectomy, especially in case of negative appendectomy (RR 1.91), largely dominated by McGory et al [6]. The possible cause of increased morbidity in negative laparoscopy is uncertain. When the diagnosis is uncertain and there are clinical findings, ultrasound or magnetic resonance imaging is recommended to establish an accurate diagnosis [7]. As to surgical procedure, in the SAGES guideline, laparoscopic appendectomy is the treatment of choice for pregnant patients with acute appendicitis [8]. The laparoscopic approach is the preferred treatment for pregnant patients with acute appendicitis, due to the shorter operative time and length of stay, and fewer complications [9]. The laparoscopic management of appendicitis during pregnancy can be performed with minimal fetal and maternal morbidity $[10,11]$. According to the most recent Nationwide Inpatient Sample study with 19, 926 pregnant women, 1,018 adverse obstetrical events were recorded in 953 pregnant women (4.8\%). Open surgery is associated with 3 times increased risk of an adverse obstetrical outcome [12]. Laparoscopic appendectomy during pregnancy is the preferred treatment, although the laparoscopic approach needs to be used with caution, particularly during the third trimester where there are greater technical challenges. It is very important not to hesitate to convert to open surgery. Maternal intra-operative blood pressure, end tidal $\mathrm{CO} 2$ and postoperative fetal hart rate should be monitored to enhance the safety of laparoscopic surgery during pregnancy [8].

The Japan Society for Endoscopic Surgery has its own endoscopic surgical skill qualification system to enhance the surgical procedure and ensure the safety and accuracy of laparoscopic surgery [13]. 


\section{Management for Complicated Appendicitis}

\section{Immediate laparoscopic surgery performed by an experi- enced surgeon is the optimal treatment for appendiceal ab- scesses}

Appendiceal abscesses are encountered in $7 \%$ of adult patients with AA [14]. Although appendectomy has been considered a gold standard treatment for AA, the management of patients with appendiceal abscesses is still controversial. Conservative management is recommended as a first line treatment, as it is associated with lower complication rates and lower morbidity. In the systematic review and metaanalysis performed by Andersson et al, immediate surgery was associated with a higher morbidity compared with conservative treatment (OR 3.3; CI: 1.9-5.6; $p<0.001$ ), while non-surgical treatment of appendicular abscesses or phlegmons, was reported to be successful in over $90 \%$ of patients, with an overall risk of recurrence of $7.4 \%$ (CI: 3.7-11.1) and only $19.7 \%$ of cases of abscesses requiring percutaneous drainage [15]. The meta-analysis by Simillis et al [16] published in 2010 with a total of 1,572 patients ( 847 treated with conservative treatment and 725 with appendectomy), conservative treatment was associated with significantly less overall complications (wound infections, abdominal/pelvic abscesses, ileus/bowel obstructions, and re-operations) compared with immediate appendectomy. There were no significant differences in the duration of the first hospitalization compared with the overall hospital stays and the duration of intravenous antibiotics. In the first prospective randomized trial by Mentula et al [17], the outcome of immediate laparoscopic surgery (30 patients) was compared with that of conservative management (30 patients) for appendiceal abscess. These results showed there was no difference in hospital stay between these groups. Although patients in the laparoscopy group had a $10 \%$ risk for bowel resection and a 13\% risk for incomplete appendectomy, there were significantly fewer patients with unplanned readmissions in the laparoscopy group: 1 (3\%) versus $8(27 \%)$, $p=0.026$. Additional interventions were required in $2(7 \%)$ patients in the laparoscopy group (percutaneous drainage) and in $9(30 \%)$ patients in the conservative group (surgery), $p=0.042$. Recurrent abscesses and failure to respond to conservative treatment were the main reasons for additional interventions. Open surgery was required in $3(10 \%)$ patients in the laparoscopy group and in $4(13 \%)$ patients in the conservative group. Postoperative complications occurred in 3 patients in the laparoscopic group versus 2 patients in the conservative group. The rate of uneventful recovery was $90 \%$ in the laparoscopy group versus $50 \%$ in the conservative group ( $p=0.002$ ). The authors concluded that immediate laparoscopic surgery in experienced hands was safe, and was a recommended first-line treatment for appendiceal abscess. A laparoscopic approach should be considered depending on the surgeon's surgical skills and experience to ensure the safety of the patients.

\section{Appendiceal neoplasms related to appendiceal abscess}

When appendiceal abscesses are encountered, the possible underlying disorders, appendicular neoplasms or Crohn's disease should be considered. The non-operative management for appendiceal abscesses has potential risks if the initial treatment is delayed for these underlying disorders. The frequency of neoplasia in adults with appendiceal mass was reported in only $1.4 \%$ [15]. In a retrospective study by Carpenter et al, in 315 patients with AA, 18 out of 24 patients with complicated appendicitis ( $7.6 \%$ of the total series) that were treated conservatively, underwent interval appendectomy. The incidence of neoplasms was significantly higher in the patients that underwent interval appendectomy than in the immediate appendectomy group (5 patients, $28 \%$ versus 3 patients, $1 \%, p<0.001)$. It remains controversial whether routine interval appendectomy is necessary. This may avoid the risk of recurrence, and also rule out the possibility of appendicular neoplasia. In addition, colonoscopy following interval appendectomy should be performed especially in patients older than 40 years.

\section{Conclusion}

Spontaneous resolution of uncomplicated AA may be common for early uncomplicated appendicitis. Active observation is required in order not to delay diagnosis, particularly for recurrent acute appendicitis. The first line treatment for acute appendicitis in pregnancy should be surgery. A laparoscopic approach is preferred for pregnant women however, when there are technical difficulties, surgeons should not hesitate to convert to open surgery. An immediate laparoscopic approach for appendiceal abscesses may be the optimal treatment, therefore, surgeons should receive training on laparoscopic surgical techniques. For complicated appendicitis, the possible underlying disorders should be considered, especially neoplasms or Crohn's disease.

\section{Conflicts of Interest}

The authors have no conflicts of interest to declare. 


\section{Acknowledgment}

This work has been presented at 6th Joint Scientific Congress of KSACS and JSACS. Session: JSACS-KSACS Joint Symposium Acute Care Surgery; Lecture Title: Acute Appendicitis; Date \& Time: April 13 (Fri), 2018, 14:50 15:10.

\section{References}

[1] Varadhan KK, Neal KR, Lobo DN. Safety and efficacy of antibiotics compared with appendicectomy for treatment of uncomplicated acute appendicitis: Meta-analysis of randomised controlled trials. BMJ 2012;344:e2156.

[2] Park HC, Kim MJ, Lee BH. Randomized clinical trial of antibiotic therapy for uncomplicated appendicitis Br J Surg 2017;104(13):1785-90.

[3] Andersen B, Nielsen TF. Appendicitis in pregnancy: diagnosis, management and complications. Acta Obstet Gynecol Scand 1999;78(9):758-62.

[4] Abbasi N, Patenaude V, Abenhaim HA. Management and outcomes of acute appendicitis in pregnancy-population-based study of over 7000 cases. BJOG 2014;121(12):1509-14.

[5] Wilasrusmee C, Sukrat B, McEvoy M, Attia J, Thakkinstian A. Systematic review and meta-analysis of safety of laparoscopic versus open appendicectomy for suspected appendicitis in pregnancy. Br J Surg 2012 ;99(11):1470-8.

[6] McGory ML, Zingmond DS, Tillou A, Hiatt JR, Ko CY, Cryer HM. Negative appendectomy in pregnant women is associated with a substantial risk of fetal loss. J Am Coll Surg 2007;205(4):534-40.

[7] Rapp EJ, Naim F, Kadivar K, Davarpanah A, Cornfeld D. Integrating MR imaging into the clinical workup of pregnant patients suspected of having appendicitis is associated with a lower negative laparotomy rate: singleinstitution study. Radiology 2013;267(1):137-44.
[8] Pearl JP, Price RR, Tonkin AE, Richardson WS, Stefanidis D [Internet]. Guidelines for diagnosis, treatment and use of laparoscopy for surgical problems during pregnancy. 2017. Available from: https://www.sages.org/ publications/guidelines/guidelines-for-diagnosis-treatment-and-use-oflaparoscopy-for-surgical-problems-during-pregnancy/.

[9] Cox TC, Huntington CR, Blair LJ, Prasad T, Lincourt AE, Augenstein VA, et al. Laparoscopic appendectomy and cholecystectomy versus open: a study in 1999 pregnant patients. Surg Endosc 2016;30(2):593-602.

[10] Affleck DG, Handrahan DL, Egger MJ, Price RR. The laparoscopic management of appendicitis and cholelithiasis during pregnancy. Am J Surg 1999;178(6):523-9.

[11] Rollins MD, Chan KJ, Price R. Laparoscopy for appendicitis and cholelithiasis during pregnancy: a new standard of care. Surg Endosc 2004;18(2):237-41.

[12] Sachs A, Guglielminotti J, Miller R, Landau R, Smiley R, Li G. Risk Factors and Risk Stratification for Adverse Obstetrical Outcomes after Appendectomy or Cholecystectomy During Pregnancy. JAMA Surg 2017;152(5):436-41.

[13] Japan Society for Endscopic Surgery [Internet]. Available from: http:// www.jses.or.jp/en/index.html.

[14] Oliak D, Yamini D, Udani VM, Lewis RJ, Arnell T, Vargas H, et al. Initial nonoperative management for periappendiceal abscess. Dis Colon Rectum 2001;44(7):936-41.

[15] Andersson RE, Petzold MG. Nonsurgical treatment of appendiceal abscess or phlegmon: a systematic review and meta-analysis. Ann Surg 2007;246(5):741-8.

[16] Simillis C, Symeonides P, Shorthouse AJ, Tekkis PP. A meta-analysis comparing conservative treatment versus acute appendectomy for complicated appendicitis (abscess or phlegmon). Surgery 2010;147 (6):818-29.

[17] Mentula P, Sammalkorpi H, Leppäniemi A. Laparoscopic Surgery or Conservative Treatment for Appendiceal Abscess in Adults? A Randomized Controlled Trial. Ann Surg 2015;262(2):237-42. 